\title{
Signatures of nuclear deformation in beta decay patterns
}

\author{
O. Moreno, P. Sarriguren, R. Álvarez-Rodríguez, E. Moya de Guerra
}

Instituto de Estructura de la Materia, Consejo Superior de Investigaciones Científicas, Serrano 123, E-28006 Madrid, Spain

October 28, 2005

\begin{abstract}
The influence of the nuclear shape on Gamow-Teller strength distributions is considered here for the case of neutron-deficient lead isotopes with even mass number from 184 to 194 . Our theoretical framework is based on a deformed Hartree-Fock (HF) mean field with pairing interactions within Bardeen-Cooper-Schrieffer approximation (BCS). Residual spin-isospin interactions in the particle-hole and particle-particle channels are also included within quasiparticle random phase approximation (QRPA). After considering the sensitivity of the Gamow-Teller (GT) strength distributions to the various ingredients in the formalism, we conclude that the $\beta^{+}$-decay patterns of these isotopes show specific signatures for each nuclear shape.
\end{abstract}

\section{Introduction and theoretical framework}

The phenomenon of nuclear shape coexistence is especially prominent in the neutron-deficient lead region. For instance, there is experimental evidence of two excited $0^{+}$states in ${ }^{186} \mathrm{~Pb}$ which correspond, together with the ground state, to three different macroscopic shapes within an energy range as small as $700 \mathrm{keV}$ [1]. The fact that the isotopes in this region undergo $\beta^{+}$-decay leads to consider the influence of the nuclear shape on their beta decay patterns. This influence has been a subject of recent theoretical and experimental interest [2][3], and it is also the topic of the present work.

The single particle energies, occupation probabilities and wave functions of the nuclei under consideration are generated from an axially-deformed Hartree-Fock mean field with effective two-body Skyrme interactions which includes pairing correlations within BCS approximation. Different Skyrme (Sk3, SG2, SLy4) and pairing forces (fixed gap or fixed strength) have been used for the sake of comparison. Gamow-Teller excitations are described within QRPA adding a spin-isospin residual interaction in the particle-hole $(p h)$ and in the particle-particle $(p p)$ channels.

\section{Results and conclusions}

For each lead isotope under study we obtain the HF energies as a function of the quadrupole deformation parameter $\beta$. Fig. 1 shows these energy-deformation curves for Sk3 Skyrme force and fixed gap pairing correlations. Prolate, spherical and oblate solutions (corresponding to the minima of the curves 


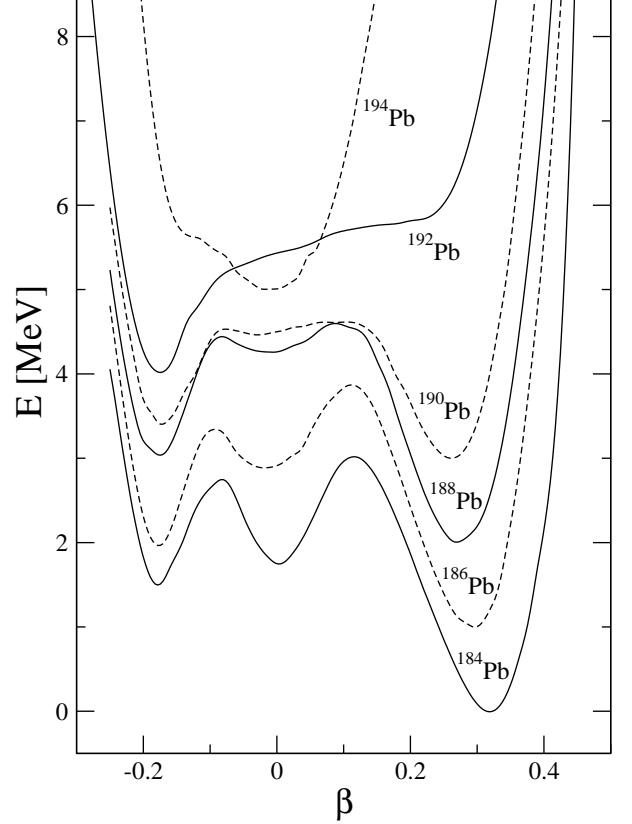

Figure 1: HF energy as a function of the quadrupole deformation $\beta$ (Sk3, fixed gap).

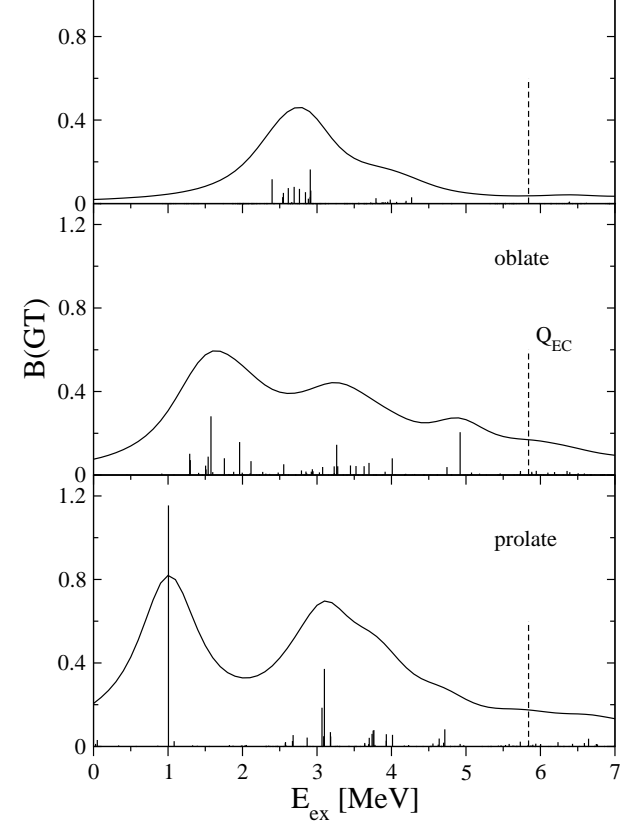

Figure 2: GT strength distributions for the equilibrium shapes of ${ }^{184} \mathrm{~Pb}$ (Sk3, fixed gap).

with deformation parameter values $\beta>0, \beta=0$, and $\beta<0$ respectively) coexist in the isotopes with mass numbers from 184 to 190. Using different Skyrme forces (Sk3, SG2, SLy4) and pairing gap or strength values, we observe that the deformation minima remain at about the same deformation values, although the relative energies may change.

The Gamow-Teller strength distributions calculated at each equilibrium deformation show specific signatures [4]. As an example we can see in Fig. 2 the case of ${ }^{184} \mathrm{~Pb}$; there is a bump between 2.5 and 3 $\mathrm{MeV}$ in the case of spherical shape, three bumps at 1.5, 3 and $5 \mathrm{MeV}$ for the oblate shape and a strong single peak at $1 \mathrm{MeV}$ and a more fragmented one at $3 \mathrm{MeV}$ for the prolate shape. These signatures turn out to be in all the cases quite stable against changes of the Skyrme and pairing forces. We have finally calculated the $\beta^{+} / E C$ half-lives for every equilibrium shape finding clear differences among them, and reaching reasonable agreement with the experimental half-lives when considering the deformed shapes.

In conclusion, we find that Gamow-Teller strength distributions and half-lives show strong dependence on nuclear deformation with specific signatures that prevail against changes in Skyrme or pairing forces. These signatures could be used in turn to identify the shape of a $\beta$-decaying nucleus.

This work was supported by Ministerio de Educación y Ciencia (Spain) under contract number BFM2002-03562.

\section{References}

[1] A. N. Andreyev et al., Nature 405 (2000) 430 .

[2] P. Sarriguren, E. Moya de Guerra and A. Escuderos, Nucl. Phys. A 13 (1999) 658.

[3] E. Nácher et al., Phys. Rev. Lett. 92 (2004) 232501.

[4] P. Sarriguren, O. Moreno, R. Álvarez-Rodríguez, E. Moya de Guerra, Phys. Rev. C, in press. 